\title{
Effects of valsartan and amlodipine on oxidative stress in type 2 diabetic patients with hypertension: a randomized, multicenter study
}

Hae Jin Kim ${ }^{1}$, Seung Jin $\mathrm{Han}^{1}$, Dae Jung Kim¹, Hak Chul Jang ${ }^{2}$, Soo Lim², Sung Hee Choi², Yong Hyun $\mathrm{Kim}^{3}$, Dong Hyun Shin${ }^{3}$, Se Hwa Kim${ }^{4}$, Tae Ho Kim ${ }^{5}$ Yu Bae Ahn ${ }^{6}$, Seung Hyun Ko ${ }^{6}$, Nan Hee Kim ${ }^{7}$, Ji A Seo ${ }^{7}$, Ha Young Kim ${ }^{8}$, and Kwan Woo Lee ${ }^{1}$

\begin{abstract}
${ }^{1}$ Department of Endocrinology and Metabolism, Ajou University School of Medicine, Suwon;

${ }^{2}$ Department of Internal Medicine, Seoul National University Bundang Hospital, Seongnam; ${ }^{3}$ Department of Internal Medicine, Daejin Medical Center, Bundang Jesaeng Hospital, Seongnam; ${ }^{4}$ Department of Internal Medicine, International St. Mary's Hospital, Catholic Kwandong University College of Medicine, Incheon; ${ }^{5}$ Department of Internal Medicine, Seoul Medical Center, Seoul; ${ }^{6}$ Department of Internal Medicine, College of Medicine, St. Vincent's Hospital, The Catholic University of Korea, Suwon; ${ }^{7}$ Department of Internal Medicine, Korea University Ansan Hospital, Ansan; ${ }^{8}$ Department of Internal Medicine, Wonkwang University Sanbon Hospital, Gunpo, Korea
\end{abstract}

Received: December 17, 2015

Revised : May 27, 2016

Accepted: May 29, 2016
Background/Aims: Oxidative stress plays an important role in the pathogenesis and progression of diabetic complications and antagonists of renin-angiotensin system and amlodipine have been reported previously to reduce oxidative stress. In this study, we compared the changes in oxidative stress markers after valsartan and amlodipine treatment in type 2 diabetic patients with hypertension and compared the changes in metabolic parameters.

Methods: Type 2 diabetic subjects with hypertension 30 to 80 years of age who were not taking antihypertensive drugs were randomized into either valsartan (n $=33)$ or amlodipine $(n=35)$ groups and treated for 24 weeks. We measured serum nitrotyrosine levels as an oxidative stress marker. Metabolic parameters including serum glucose, insulin, lipid profile, and urine albumin and creatinine were also measured.

Results: After 24 weeks of valsartan or amlodipine treatment, systolic and diastolic blood pressure decreased, with no significant difference between the groups. Both groups showed a decrease in serum nitrotyrosine $(7.74 \pm 7.30 \mathrm{nmol} / \mathrm{L}$ vs. $3.95 \pm 4.07$ $\mathrm{nmol} / \mathrm{L}$ in the valsartan group and $8.37 \pm 8.75 \mathrm{nmol} / \mathrm{L}$ vs. $2.68 \pm 2.23 \mathrm{nmol} / \mathrm{L}$ in the amlodipine group) with no significant difference between the groups. Other parameters including glucose, lipid profile, albumin-to-creatinine ratio, and homeostasis model assessment of insulin resistance showed no significant differences before and after treatment in either group.

Conclusions: Valsartan and amlodipine reduced the oxidative stress marker in type 2 diabetic patients with hypertension.

Keywords: Valsartan; Amlodipine; Diabetes mellitus, type 2; Oxidative stress

\section{Correspondence to Kwan Woo Lee, M.D.}

Department of Endocrinology and Metabolism, Ajou University School of Medicine, 164 World cup-ro, Yeongtong-gu, Suwon 16499, Korea

Tel: $+82-31-219-4526$

Fax: +82-31-219-4497

E-mail:1kw65@ajou.ac.kr 


\section{INTRODUCTION}

Cardiovascular disease is a leading cause of premature mortality in patients with diabetes mellitus [1]. The risk of premature mortality increases in diabetic patients with hypertension [2]. The proper treatment and control of blood pressure (BP) is important to prevent macrovascular complications and reduce the progression of microvascular complications.

The renin-angiotensin-aldosterone system (RAAS) is considered a possible unifying pathophysiology of diabetes and hypertension and associated complications. The first choice of drugs for treatment of hypertension in diabetic patients are angiotensin-converting-enzyme inhibitors (ACEIs) or angiotensin II receptor blockers (ARBs) due to the reduction in macrovascular and microvascular complications and their positive metabolic effects, which are independent of BP-lowering effects [3].

Oxidative stress is defined as excessive production of reactive oxygen species (ROS) in the presence of diminished anti-oxidant substances, and it has emerged as an important mechanism for the development of diabetic complications [4]. The metabolic abnormalities of diabetes cause mitochondrial superoxide overproduction in endothelial cells of both large and small vessels, as well as in the myocardium, which results in the activation of major pathways involved in the pathogenesis of complications [5]. Oxidative stress has also been suggested to be involved in the pathogenesis of target organ damage in hypertension [6]. Angiotensin II is known as an inducer of oxidative stress in cardiovascular tissue [7] and it is suggested that RAAS antagonists such as ACEI or ARB can reduce inflammation and oxidative stress $[8,9]$.

Amlodipine is a dihydropyridine calcium channel blocker (CCB), which has also displayed antioxidant effects in in vitro and in vivo studies, independent of its calcium channel blocking action [10]. Amlodipine is commonly used due to its ability to assist patients with diabetes reach their target $\mathrm{BP}$ value [11].

In this study, we compared the changes in an oxidative stress marker after valsartan and amlodipine treatment in type 2 diabetic patients with hypertension and compared the changes in metabolic parameters.

\section{METHODS}

\section{Subjects}

Type 2 diabetic patients who visited the endocrinology outpatient clinic at seven general hospitals in Korea were enrolled in this study. The patients met the inclusion criteria, which consisted of (1) systolic BP (SBP) 135 to $160 \mathrm{mmHg}$ and/or diastolic BP (DBP) 85 to $100 \mathrm{mmHg}$; (2) 30 to 80 years of age; (3) not taking antihypertensive drugs within 2 weeks prior to screening date; and (4) glycated hemoglobin ( $\mathrm{HbArc}) \leq 9.0 \%$. Patients with secondary hypertension, impaired hepatic function (defined by serum levels of either alanine transaminase or aspartate transaminase $>2.5$ times the upper limit of normal), renal impairment (serum creatinine $>2.0 \mathrm{mg} / \mathrm{dL}$ ), or any other clinically relevant serious disease, including malignancy, serious infection, heart failure, myocardial infarction or stroke within 6 months prior to the screening date, or patients who were pregnant, breast-feeding, or planning to bear a child were excluded. Due to possible effects on oxidative stress markers, patients who consumed statin, thioctic acid or thiazolidinedione within 3 months or systemic steroids or herbal medications within 1 month prior to the screening date were excluded. Patients taking antiplatelet drugs, vitamins, or estrogen at the initial screening date were included but were not allowed to adjust the drug dosages. Antidiabetic medications were allowed to be adjusted throughout the course of the study, with the exception of thiazolidinedione.

This study was approved by all Institutional Review Boards of the concerned hospitals, and all enrolled subjects provided written informed consent forms. This trial was registered in Clinical Research Information Service, Republic of Korea (registration number KCTooo1498).

\section{Study design and treatment}

This was a randomized, open label, active control, multicenter study. The subjects were randomized using a computer-generated allocation system into groups for treatment with either amlodipine $5 \mathrm{mg}$ or valsartan 80 mg every morning for 24 weeks. BP was measured at baseline and 8, 16, and 24 weeks. SBP and DBP were measured 10 minutes after arriving at the clinics and another 5 minutes later, and the average of these two 
times were recorded. If the BP had not reached either $\mathrm{SBP}<130 \mathrm{mmHg}$ or DBP $<80 \mathrm{mmHg}$ at 8 weeks of treatment, the dosage of each drug was doubled. If the subjects' BP in both groups did not reach the target BP (130/80 $\mathrm{mmHg}$ ) at 16 weeks, thiazide $12.5 \mathrm{mg}$ was additionally prescribed, as it had been previously proven to have no effect on oxidative stress [12]. Blood and urine were sampled in the morning after overnight fasting at baseline and after 24 weeks of treatment with each drug.

\section{Biochemical measurements}

The blood samples were centrifuged and the resulting supernatants as well as urine samples were stored at $-70^{\circ} \mathrm{C}$ until analyses. Glucose, HbArc, insulin, total cholesterol, triglyceride, and high density lipoprotein cholesterol concentrations were measured using standard methods. The low density lipoprotein cholesterol concentration was calculated using the Friedewald equation [13]. Homeostasis model assessment of insulin resistance (HOMA-IR) [(fasting insulin $[\mu \mathrm{U} / \mathrm{mL}] \times$ fasting glucose [mmol/L]) / 22.5)] was calculated as an index of insulin resistance. We measured serum nitrotyrosine as an oxidative stress marker using an ELISA kit (Cell Biolabs, San Diego, CA, USA), and the intra- and interassay coefficients of variation were $6 \%$ and $10 \%$, respectively. Serum high-sensitive C-reactive protein (hsCRP, Denka-seiken, Tokyo, Japan) and urine albumin and creatinine were also measured.

\section{Statistical analyses}

Statistical analyses were performed using the IBM SPSS Statistics version 23.0 (IBM Co., Armonk, NY, USA). Nitrotyrosine was log transformed, resulting in normal distributions. We used paired $t$ tests to analyze the differences in values before and after 24 weeks of valsartan or amlodipine administration. Parameters of each group at baseline and 24 weeks after administration were compared using Student unpaired $t$ tests. Differences between each group were also compared at baseline and 24 weeks using Student unpaired $t$ tests. A $p$ value $<0.05$ was considered to indicate statistical significance.

\section{RESULTS}

Sixty-eight subjects were enrolled and 58 subjects completed the study. The baseline characteristics of valsartan and amlodipine groups are shown in Table 1. The mean age of the subjects was 53.2 and 55.8 years and the mean diabetes duration was 7.2 and 8.9 years, respectively. The mean SBP and DBP were 146.1 and $89.8 \mathrm{mmHg}$ in the valsartan group and 147.1 and 91.0 $\mathrm{mmHg}$ in the amlodipine group. The mean body mass index (BMI) was 25.3 and $24.6 \mathrm{~kg} / \mathrm{m}^{2}$ and the mean HbAic was $7.3 \%$ and $7.1 \%$, respectively. There were no significant differences between the groups in BP, glucose, lipid profiles, BMI and the antidiabetic medication. During the study, one more class of antidiabetic medication was added in two subjects from the valsartan group and two subjects from amlodipine group.

At the conclusion of the study at 24 weeks, the number of subjects who took valsartan in doses of 80,160 , and 160 mg plus thiazide $12.5 \mathrm{mg}$ was 17,8 , and 6 , respectively, in the valsartan group. In the amlodipine group, 18 subjects took $5 \mathrm{mg}$ and nine took $10 \mathrm{mg}$ of amlodipine. At week 24, target BP (130/80 mmHg) was not reached in seven subjects from the valsartan group and seven subjects from the amlodipine group. After 24 weeks of valsartan or amlodipine treatment, SBP and DBP were decreased in both groups but the difference of decrease between the groups was not significant (Tables 2 and 3). The serum level of nitrotyrosine, an oxidative stress marker, decreased after 24 weeks of valsartan treatment compared to baseline (log nitrotyrosine $0.84 \pm 0.39 \mathrm{nmol} / \mathrm{L}$ vs. $0.51 \pm 0.39 \mathrm{nmol} / \mathrm{L}$, $p=0.007)$ and decreased after amlodipine treatment (log nitrotyrosine $0.68 \pm 0.59 \mathrm{nmol} / \mathrm{L}$ vs. $0.38 \pm 0.39 \mathrm{nmol} / \mathrm{L}, p=$ 0.037)(Table 2, Fig. 1). The decrease in serum nitrotyrosine was not significantly different between the two groups (Table 3). There was also no significant difference in the decrease in serum nitrotyrosine between both groups, even after dividing the subjects based on albuminuria levels (urine albumin to creatinine ratio $[\mathrm{ACR}]<30$ and $\geq$ $30 \mu \mathrm{g} / \mathrm{mg} \mathrm{Cr}$ ). Other parameters including glucose, lipid profile, urine ACR, hsCRP, and HOMA-IR showed no significant differences before and after treatment in either group (Table 2). The decrease in the oxidative stress marker did not correlate with decreased BP or urine albumin excretion in each group (data not shown). 
Table 1. Subject baseline characteristics and metabolic parameters

\begin{tabular}{|c|c|c|c|}
\hline Characteristic & Valsartan $(\mathrm{n}=33)$ & Amlodipine $(\mathrm{n}=35)$ & $p$ value $^{\mathrm{a}}$ \\
\hline Age, yr & $53.2 \pm 9.5$ & $55.8 \pm 10.1$ & 0.281 \\
\hline Sex, male/female & $23 / 10$ & $25 / 10$ & 0.878 \\
\hline DM duration, yr & $7 \cdot 2 \pm 5 \cdot 9$ & $8.9 \pm 7.0$ & 0.281 \\
\hline $\mathrm{SBP}, \mathrm{mmHg}$ & $146.1 \pm 7.9$ & $147 \cdot 1 \pm 7 \cdot 4$ & 0.594 \\
\hline $\mathrm{DBP}, \mathrm{mmHg}$ & $89.8 \pm 7.0$ & $91.0 \pm 7.3$ & 0.489 \\
\hline Body weight, kg & $68.9 \pm 9.2$ & $68.1 \pm 10.3$ & 0.754 \\
\hline $\mathrm{BMI}, \mathrm{kg} / \mathrm{m}^{2}$ & $25 \cdot 3 \pm 2.8$ & $24.6 \pm 2.4$ & 0.214 \\
\hline Waist, cm & $89.7 \pm 6.7$ & $88.5 \pm 5.7$ & 0.438 \\
\hline Total cholesterol, mg/dL & $185.2 \pm 26.4$ & $183.8 \pm 30.8$ & 0.837 \\
\hline Triglycerides, mg/dL & $157.2 \pm 93.6$ & $175.2 \pm 125.2$ & 0.505 \\
\hline LDL-C, mg/dL & $106.3 \pm 27 \cdot 3$ & $102.0 \pm 30.4$ & 0.545 \\
\hline HDL-C, mg/dL & $47.1 \pm 7.8$ & $46.4 \pm 11.1$ & 0.769 \\
\hline Glucose, mg/dL & $137.7 \pm 28.7$ & $140.4 \pm 28.5$ & 0.702 \\
\hline $\mathrm{HbAlc}, \%$ & $7 \cdot 3 \pm 0.8$ & $7.1 \pm 0.8$ & 0.419 \\
\hline Insulin, $\mu \mathrm{U} / \mathrm{mL}$ & $14.83 \pm 29.81$ & $11.24 \pm 10.60$ & 0.511 \\
\hline HOMA-IR & $5.26 \pm 12.50$ & $3.63 \pm 3.03$ & 0.469 \\
\hline Smoking, \% & 36.4 & 20.0 & 0.193 \\
\hline \multicolumn{4}{|l|}{ Antidiabetic medications, \% } \\
\hline Sulfonylurea & 51.5 & 48.6 & 0.808 \\
\hline Metformin & 78.8 & 80.0 & 0.902 \\
\hline DPP-4 inhibitor & 21.2 & 37.1 & 0.150 \\
\hline Meglitinide & 3.0 & 2.9 & 0.966 \\
\hline$\alpha$-Glycosidase inhibitor & 9.1 & $5 \cdot 7$ & 0.594 \\
\hline Insulin & 0 & 8.6 & 0.085 \\
\hline
\end{tabular}

Values are presented as mean $\pm \mathrm{SD}$.

DM, diabetes mellitus; SBP, systolic blood pressure; DBP, diastolic blood pressure; BMI, body mass index; LDL-C, low density lipoprotein cholesterol; HDL-C, high density lipoprotein cholesterol; HbA1c, glycated hemoglobin; HOMA-IR, homeostasis model assessment of insulin resistance; DPP-4, dipeptidyl peptidase 4 .

${ }^{a} p$ between valsartan and amlodipine groups using an independent $t$ test.

\section{DISCUSSION}

The present study showed valsartan and amlodipine reduced the oxidative stress marker serum nitrotyrosine in type 2 diabetic patients with hypertension, showing no correlation with their BP lowering effect.

The importance of oxidative stress as a pivotal mechanism for the development of diabetic complications has been highly recognized [4,5]. Stimuli such as hyperglycemia or high BP increase ROS production, such as hydrogen peroxide $\left(\mathrm{H}_{2} \mathrm{O}_{2}\right)$, peroxynitrite and other free radicals [14]. An increase in oxidative/nitrosative stress describes cellular antioxidant defenses that are inade- quate to fully inactivate the ROS and reactive nitrogen species (RNS), resulting in damage to DNA, lipids, and proteins. Measuring cellular products of oxidative damage has been suggested as a method to evaluate the degree of oxidative stress. RNS such as peroxynitrite anion and nitrogen dioxide mediate tyrosine nitration, which represents a post-translational modification of proteins in situations of high oxidative stress $[15,16]$. The association of nitrotyrosine with cardiovascular diseases was reported in previous human studies [15].

Angiotensin II acts as a ROS inducer via an nicotinamide adenine dinucleotide phosphate-dependent mechanism, increasing ROS formation in the peripheral circu- 
Table 2. Metabolic parameters before and after 24 weeks of treatment with valsartan or amlodipine

\begin{tabular}{|c|c|c|c|c|c|c|c|c|}
\hline \multirow{2}{*}{ Parameter } & \multicolumn{3}{|c|}{$\operatorname{Valsartan}(\mathrm{n}=31)$} & \multicolumn{3}{|c|}{ Amlodipine $(\mathrm{n}=27)$} & \multirow{2}{*}{$p$ value $^{c}$} & \multirow{2}{*}{$p$ value $^{\mathrm{d}}$} \\
\hline & Baseline & 24 Weeks & $p$ value $^{a}$ & Baseline & 24 Weeks & $p$ value $^{\mathrm{b}}$ & & \\
\hline $\mathrm{SBP}, \mathrm{mmHg}$ & $145.8 \pm 8.0$ & $129.3 \pm 10.1$ & $<0.001$ & $146.9 \pm 7.1$ & $130.7 \pm 11.0$ & $<0.001$ & 0.587 & 0.627 \\
\hline $\mathrm{DBP}, \mathrm{mmHg}$ & $89.2 \pm 7.2$ & $79.9 \pm 7.2$ & $<0.001$ & $92.0 \pm 7.7$ & $79.8 \pm 7.8$ & $<0.001$ & 0.169 & 0.956 \\
\hline Body weight, kg & $68.6 \pm 9.5$ & $68.3 \pm 9.5$ & 0.363 & $69.6 \pm 9.8$ & $69.0 \pm 9.9$ & 0.181 & 0.933 & 0.777 \\
\hline $\mathrm{BMI}, \mathrm{kg} / \mathrm{m}^{2}$ & $25.4 \pm 2.9$ & $25 \cdot 3 \pm 2.9$ & 0.374 & $24.4 \pm 2.3$ & $24.2 \pm 2.3$ & 0.158 & 0.142 & 0.156 \\
\hline Waist, cm & $89.6 \pm 7.1$ & $87.4 \pm 17 \cdot 3$ & 0.461 & $88.7 \pm 5.3$ & $87.8 \pm 5.8$ & 0.120 & 0.456 & 0.927 \\
\hline $\mathrm{TC}, \mathrm{mg} / \mathrm{dL}$ & $185.1 \pm 27.7$ & $182.5 \pm 32.2$ & 0.601 & $182.2 \pm 33.2$ & $179.4 \pm 28.8$ & 0.612 & 0.731 & 0.711 \\
\hline $\mathrm{TG}, \mathrm{mg} / \mathrm{dL}$ & $143.5 \pm 78.9$ & $163.6 \pm 91.0$ & 0.224 & $174.2 \pm 126.9$ & $159.8 \pm 108.4$ & 0.427 & 0.292 & 0.889 \\
\hline LDL-C, mg/dL & $108.1 \pm 27.0$ & $103.7 \pm 32.1$ & 0.436 & $104.1 \pm 33 \cdot 3$ & $102.3 \pm 29.7$ & 0.767 & 0.605 & 0.867 \\
\hline HDL-C, mg/dL & $47 \cdot 9 \pm 7.8$ & $47 \cdot 1 \pm 9.7$ & 0.544 & $44.9 \pm 11.1$ & $46.4 \pm 11.9$ & 0.329 & 0.328 & 0.821 \\
\hline Glucose, mg/dL & $138.2 \pm 29.0$ & $144.7 \pm 45 \cdot 7$ & 0.304 & $145.2 \pm 30.6$ & $135.0 \pm 29.1$ & 0.061 & 0.384 & 0.369 \\
\hline $\mathrm{HbAic}, \%$ & $7.2 \pm 0.8$ & $7.3 \pm 0.9$ & 0.847 & $7.1 \pm 0.8$ & $6.8 \pm 1.8$ & 0.308 & 0.792 & 0.276 \\
\hline Insulin, $\mu \mathrm{U} / \mathrm{mL}$ & $14.27 \pm 30.29$ & $13.73 \pm 26.82$ & 0.528 & $10.66 \pm 11.81$ & $11.18 \pm 15.76$ & 0.651 & 0.486 & 0.677 \\
\hline HOMA-IR & $5.66 \pm 13.46$ & $5 \cdot 50 \pm 11.40$ & 0.734 & $3.57 \pm 3.40$ & $3.89 \pm 5.94$ & 0.640 & 0.466 & 0.544 \\
\hline hsCRP, mg/L & $1.51 \pm 1.61$ & $1.86 \pm 2.07$ & 0.288 & $3.04 \pm 9.02$ & $1.22 \pm 1.51$ & 0.284 & 0.417 & 0.206 \\
\hline $\mathrm{ACR}, \mu \mathrm{g} / \mathrm{mg} \mathrm{Cr}$ & $39 \cdot 3 \pm 70.6$ & $29.6 \pm 51.5$ & 0.185 & $41.0 \pm 67.0$ & $32.5 \pm 55.1$ & 0.130 & 0.699 & 0.852 \\
\hline Log nitrotyrosine, $\mathrm{nmol} / \mathrm{L}^{\mathrm{e}}$ & $0.84 \pm 0.39$ & $0.51 \pm 0.39$ & 0.007 & $0.68 \pm 0.59$ & $0.38 \pm 0.39$ & 0.037 & 0.802 & 0.206 \\
\hline
\end{tabular}

Values are presented as mean $\pm \mathrm{SD}$.

SBP, systolic blood pressure; DBP, diastolic blood pressure; BMI, body mass index; TC, total cholesterol; TG, triglycerides; LDL-C, low density lipoprotein cholesterol; HDL-C, high density lipoprotein cholesterol; HbA1c, glycated hemoglobin; HOMA-IR, homeostasis model assessment of insulin resistance; hsCRP, high-sensitive C-reactive protein; ACR, urine albumin to creatinine ratio.

${ }^{a} p$ value before and after valsartan treatment using a paired $t$ test.

${ }^{\mathrm{b}} p$ value before and after amlodipine treatment using a paired $t$ test.

${ }^{c} p$ value comparison between valsartan and amlodipine at baseline using an independent $t$ test.

$\mathrm{d}_{p}$ value comparison between valsartan and amlodipine at 24 weeks using an independent $t$ test.

${ }^{\mathrm{e}}$ Nitrotyrosine was log transformed.

lation [17] and ACEIs or ARBs may reduce oxidative stress [18]. RAAS inhibitors were proven advantageous in the initial treatment for hypertension in diabetic patients. Although evidence of the advantages of RAAS inhibitors on cardiovascular diseases outcomes in diabetic patients is conflicting, it is recommended as the initial treatment for hypertension [19].

Several previously published papers support our findings that $A R B$ reduces oxidative stress. In a Japanese study, subjects with essential hypertension assigned to receive the ARB candesartan, or other antihypertensive agents that do not block the RAAS showed reduction of oxidative stress and inflammation in the candesartan group [20]. Valsartan also reduced oxidative stress in estrogen-deficient rats, an endothelial dysfunction model
[21] and in rat models of myocardial ischemia-reperfusion [22]. Another study reported that valsartan treatment reduced renal inflammation and oxidative stress markers and slowed the progression of diabetic nephropathy in obese and diabetic mice [23].

CCBs are a chemically heterogeneous group blocking the transfer of calcium ions across cell membranes; thereby, reducing intracellular calcium. Amlodipine is a dihydropyridine CCB that is more selective in blocking L-type calcium channels in vascular smooth muscle cells, inducing vascular relaxation and reduced BP [24]. Previous studies reported that amlodipine, a highly lipophilic CCB, inhibits oxidative damage to lipids associated with cellular membranes and lipoprotein particles [10]. Amlodipine inhibited lipid peroxide forma- 
Table 3. Changes $(\Delta)$ in metabolic parameters after 24 weeks of treatment with valsartan or amlodipine

\begin{tabular}{lccc}
\hline Parameter & Valsartan & Amlodipine & $p^{\text {value }}{ }^{\mathrm{a}}$ \\
\hline$\Delta$ SBP & $-16.5 \pm 12.6$ & $-16.2 \pm 13.3$ & 0.938 \\
$\Delta$ DBP & $-9.3 \pm 9.4$ & $-12.1 \pm 8.3$ & 0.228 \\
$\Delta$ Waist & $-2.2 \pm 15.5$ & $-0.9 \pm 2.8$ & 0.672 \\
$\Delta$ HbAic & $0.0 \pm 0.6$ & $-0.3 \pm 1.4$ & 0.290 \\
$\Delta$ Insulin & $-0.55 \pm 4.78$ & $0.52 \pm 5.72$ & 0.448 \\
$\Delta$ HOMA-IR & $-0.17 \pm 2.56$ & $0.312 \pm 3.16$ & 0.553 \\
$\Delta$ hsCRP & $0.35 \pm 1.78$ & $-1.82 \pm 8.33$ & 0.163 \\
$\Delta$ ACR & $-9.7 \pm 37.7$ & $-8.5 \pm 25.3$ & 0.902 \\
$\Delta$ Log nitrotyrosine ${ }^{\mathrm{b}}$ & $-0.32 \pm 0.54$ & $-0.30 \pm 0.58$ & 0.889 \\
\hline
\end{tabular}

Values are presented as mean $\pm \mathrm{SD}$.

SBP, systolic blood pressure; DBP, diastolic blood pressure; HbAic, glycated hemoglobin; HOMA-IR, homeostasis model assessment of insulin resistance; hsCRP, high-sensitive C-reactive protein; ACR, urine albumin to creatinine ratio.

${ }^{a} p$ between valsartan and amlodipine groups using an independent $t$ test.

${ }^{\mathrm{b}}$ Nitrotyrosine was log transformed.

tion, independent of calcium channel modulation and this antioxidant activity of amlodipine is attributed to both its high lipophilicity and a chemical structure that facilitates proton-donating and resonance-stabilization mechanisms that quench the free-radical reaction $[24,25]$. There was a Japanese study showing that amlodipine decreased oxidative stress in patients with type 2 diabetes [26]. Reportedly, amlodipine reduced BP in the early stages of treatment as well as the SBP variability more than atenolol or ACEI, resulting in a better protecting effect than ARBs against stroke and myocardial infarction $[27,28]$.

There have been several studies comparing ARBs and CCBs. In a study in which valsartan and amlodipine were compared in hypertensive patients with left ventricular hypertrophy, formation of ROS by monocytes was reduced in the valsartan group but not in the amlodipine group [29]. In another study evaluating whether the use of the ARB olmesartan and amlodipine improved the endothelium-dependent coronary dilation in hypertensive patients, the olmesartan group showed improvement, as well as an increase in the antioxidant marker, superoxide dismutase, independent of BP reduction [30]. A study on the effects of valsartan and amlodipine on endothelial function and oxidative stress in patients

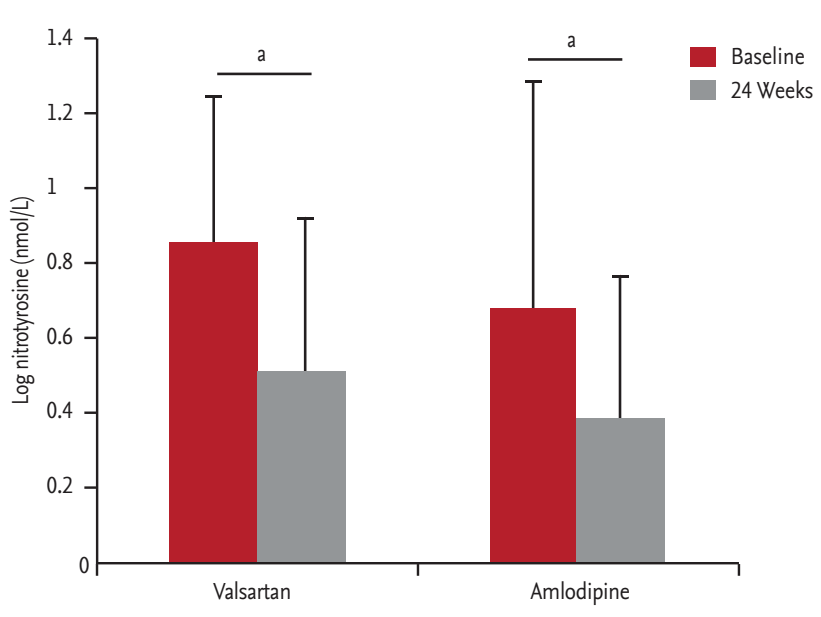

Figure 1. Levels of serum nitrotyrosine before and after 24 weeks of treatment with valsartan or amlodipine. Nitrotyrosine was log transformed. ${ }^{\mathrm{a}} \mathrm{p}<0.05$.

of essential hypertension also showed a decrease in oxidative stress markers after 1 year of treatment with valsartan but not with amlodipine [31]. However, another study on the effects of amlodipine and valsartan in endstage renal disease patients on hemodialysis showed a decrease in oxidative stress in both groups [32], similar to our results in type 2 diabetic patients with essential hypertension who did not take antihypertensive drugs.

RAAS blockers are recommended as a pharmacological therapy for diabetic patients with hypertension [19]. However, a combination of more than one antihypertensive drug is generally needed to reach the target BP [11]. In the Avoiding Cardiovascular events through Combination therapy in Patients Living with Systolic Hypertension (ACCOMPLISH) trial, it was suggested that a combination of RAAS blockers and amlodipine was superior to RAAS blockers and hydrochlorothiazide in reducing cardiovascular events among patients with hypertension and cardiovascular risk factors, with the greatest impact among diabetic patients [33]. Our results showing an improved oxidative stress marker after valsartan or amlodipine treatment can somewhat support the fact that ARB and CCB are recommended to treat diabetic patients with hypertension.

In this study, valsartan showed a decrease in serum nitrotyrosine, but not in albuminuria or hsCRP in type 2 diabetic patients with hypertension. The lower dosage of valsartan used in our study may have produced negative results, taking into consideration another re- 
port showing a higher dose of angiotensin II blockade was used to reduce albuminuria as well as inflammation [23]. Other possible reasons for this discrepancy are that the number of enrolled subjects in the present study is considered low and the standard deviation of metabolic parameters was high. Besides the low number of study subjects, not having a placebo or a control group, and not being a blind study contributed to the limitations of our study.

In conclusion, valsartan and amlodipine reduced the oxidative stress marker in type 2 diabetic patients with hypertension. Considering the role of oxidative stress on the progression of diabetic complications, either drug, or their combination, can be suitable options for treating patients with diabetes and hypertension.

\section{KEY MESSAGE}

1. Valsartan and amlodipine reduced the oxidative stress marker in type 2 diabetic patients with hypertension.

2. Valsartan, amlodipine, or their combination, can be suitable options for treating patients with diabetes and hypertension.

\section{Conflict of interest}

This study was supported by a grant from Novartis Korea Ltd. Novartis Korea Ltd. did not have any role for designing, data collection, data analysis, and writing the manuscript.

\section{REFERENCES}

1. Emerging Risk Factors Collaboration, Sarwar N, Gao P, et al. Diabetes mellitus, fasting blood glucose concentration, and risk of vascular disease: a collaborative meta-analysis of 102 prospective studies. Lancet 2010;375:2215-2222.

2. Hu G, Jousilahti P, Tuomilehto J. Joint effects of history of hypertension at baseline and type 2 diabetes at baseline and during follow-up on the risk of coronary heart disease. Eur Heart J 2007;28:3059-3066.

3. Hsueh WA, Wyne K. Renin-angiotensin-aldosterone system in diabetes and hypertension. J Clin Hypertens (Greenwich) 2011;13:224-237.
4. Araki E, Nishikawa T. Oxidative stress: a cause and therapeutic target of diabetic complications. J Diabetes Investig 2010;1:90-96.

5. Giacco F, Brownlee M. Oxidative stress and diabetic complications. Circ Res 2010;107:1058-1070.

6. Dikalov SI, Ungvari Z. Role of mitochondrial oxidative stress in hypertension. Am J Physiol Heart Circ Physiol 2013;305:H1417-H1427.

7. Chabrashvili T, Kitiyakara C, Blau J, et al. Effects of ANG II type 1 and 2 receptors on oxidative stress, renal NADPH oxidase, and SOD expression. Am J Physiol Regul Integr Comp Physiol 2003;285:R117-R124.

8. Hornig B, Landmesser U, Kohler C, et al. Comparative effect of ACE inhibition and angiotensin II type 1 receptor antagonism on bioavailability of nitric oxide in patients with coronary artery disease: role of superoxide dismutase. Circulation 2001;103:799-805.

9. Fliser D, Buchholz K, Haller H; EUropean Trial on Olmesartan and Pravastatin in Inflammation and Atherosclerosis (EUTOPIA) Investigators. Antiinflammatory effects of angiotensin II subtype 1 receptor blockade in hypertensive patients with microinflammation. Circulation 2004;110:1103-1107.

10. Mason RP. Mechanisms of plaque stabilization for the dihydropyridine calcium channel blocker amlodipine: review of the evidence. Atherosclerosis 2002;165:191-199.

11. Aksnes TA, Skarn SN, Kjeldsen SE. T reatment of hypertension in diabetes: what is the best therapeutic option? Expert Rev Cardiovasc Ther 2012;10:727-734.

12. Zhou MS, Schulman IH, Jaimes EA, Raij L. Thiazide diuretics, endothelial function, and vascular oxidative stress. J Hypertens 2008;26:494-500.

13. Friedewald WT, Levy RI, Fredrickson DS. Estimation of the concentration of low-density lipoprotein cholesterol in plasma, without use of the preparative ultracentrifuge. Clin Chem 1972;18:499-502.

14. Forstermann U. Oxidative stress in vascular disease: causes, defense mechanisms and potential therapies. Nat Clin Pract Cardiovasc Med 2008;5:338-349.

15. Ho E, Karimi Galougahi K, Liu CC, Bhindi R, Figtree GA. Biological markers of oxidative stress: applications to cardiovascular research and practice. Redox Biol 2013;1:483491.

16. Dalle-Donne I, Rossi R, Colombo R, Giustarini D, Milzani A. Biomarkers of oxidative damage in human disease. Clin Chem 2006;52:601-623. 
17. Brasier AR, Recinos A 3rd, Eledrisi MS. Vascular inflammation and the renin-angiotensin system. Arterioscler Thromb Vasc Biol 2002;22:1257-1266.

18. Shimada K, Murayama T, Yokode M, Kita T, Fujita M, Kishimoto C. Olmesartan, a novel angiotensin II type 1 receptor antagonist, reduces severity of atherosclerosis in apolipoprotein E deficient mice associated with reducing superoxide production. Nutr Metab Cardiovasc Dis 2011;21:672-678.

19. American Diabetes Association. 8. Cardiovascular disease and risk management. Diabetes Care 2016;39 Suppl 1:S6o-S71.

20. Dohi Y, Ohashi M, Sugiyama M, Takase H, Sato K, Ueda R. Candesartan reduces oxidative stress and inflammation in patients with essential hypertension. Hypertens Res 2003;26:691-697.

21. Yung LM, Wong WT, Tian XY, et al. Inhibition of renin-angiotensin system reverses endothelial dysfunction and oxidative stress in estrogen deficient rats. PLoS One 2011;6:e17437.

22. Wu B, Lin R, Dai R, Chen C, Wu H, Hong M. Valsartan attenuates oxidative stress and NF- $\mathrm{KB}$ activation and reduces myocardial apoptosis after ischemia and reperfusion. Eur J Pharmacol 2013;705:140-147.

23. Zhou G, Cheung AK, Liu X, Huang Y. Valsartan slows the progression of diabetic nephropathy in $\mathrm{db} / \mathrm{db}$ mice via a reduction in podocyte injury, and renal oxidative stress and inflammation. Clin Sci (Lond) 2014;126:707-720.

24. Mason RP, Marche P, Hintze TH. Novel vascular biology of third-generation L-type calcium channel antagonists: ancillary actions of amlodipine. Arterioscler Thromb Vasc Biol 2003;23:2155-2163.

25. Park CG. Is amlodipine more cardioprotective than other antihypertensive drug classes? Korean J Intern Med
2014;29:301-304.

26. Takebayashi K, Naruse R, Aso Y, Inukai T. The effect of amlodipine on oxidative stress in patients with type 2 diabetes. SRX Med 2010;2010:326840.

27. Julius S, Kjeldsen SE, Weber M, et al. Outcomes in hypertensive patients at high cardiovascular risk treated with regimens based on valsartan or amlodipine: the VALUE randomised trial. Lancet 2004;363:2022-2031.

28. Wang JG, Yan P, Jeffers BW. Effects of amlodipine and other classes of antihypertensive drugs on long-term blood pressure variability: evidence from randomized controlled trials. J Am Soc Hypertens 2014;8:340-349.

29. Yasunari K, Maeda K, Watanabe T, Nakamura M, Yoshikawa J, Asada A. Comparative effects of valsartan versus amlodipine on left ventricular mass and reactive oxygen species formation by monocytes in hypertensive patients with left ventricular hypertrophy. J Am Coll Cardiol 2004;43:2116-2123.

30. Naya M, Tsukamoto T, Morita K, et al. Olmesartan, but not amlodipine, improves endothelium-dependent coronary dilation in hypertensive patients. J Am Coll Cardiol 2007;50:1144-1149.

31. Hirooka Y, Kimura Y, Sagara Y, Ito K, Sunagawa K. Effects of valsartan or amlodipine on endothelial function and oxidative stress after one year follow-up in patients with essential hypertension. Clin Exp Hypertens 2008;30:267276.

32. Aslam S, Santha T, Leone A, Wilcox C. Effects of amlodipine and valsartan on oxidative stress and plasma methylarginines in end-stage renal disease patients on hemodialysis. Kidney Int 2006;70:2109-2115.

33. Weber MA, Bakris GL, Jamerson K, et al. Cardiovascular events during differing hypertension therapies in patients with diabetes. J Am Coll Cardiol 2010;56:77-85. 